\title{
Temperature dependence of self-broadening in molecular-oxygen spectrum $\left(^{*}\right)$
}

\author{
M. DE Angelis, L. Gianfrani $\left({ }^{* *}\right)$, F. Pavone $(* * *)$
}

A. SAsso and G. M. Tino

Dipartimento di Scienze Fisiche, Università di Napoli «Federico II»

INO, Sezione di Napoli - Mostra d'Oltremare, Pad. 20, 80125 Napoli, Italy

(ricevuto il 4 Dicembre 1995; approvato l'8 Gennaio 1995)

Summary. - The ${ }^{P} P(k=9)$ magnetic-dipole transition of the oxygen 0-0 vibrational band of the red system $b^{1} \Sigma_{\mathrm{g}}^{+} \leftarrow X^{3} \Sigma_{\mathrm{g}}^{-}$was observed with absorption spectroscopy using a tunable $\mathrm{CW}$ diode laser. A systematic investigation of the self-collisional broadening was performed for different gas pressures and temperatures. The temperature dependence of the self-broadening coefficient was determined to be $T^{-(1.02 \pm 0.05)}$. Our results are of interest for collisional theory and as reference data in atmospheric monitoring applications.

PACS 33.70.Jg - Line and band widths, shapes, and shifts.

PACS 33.20.Ea - Infrared spectra.

\section{1. - Introduction}

An important reason for studying pressure broadening in molecular spectra is represented by the information which may be deduced about the various types of forces involved in collisions between molecules [1].

Particularly interesting appears such a study for molecular-oxygen lines. Since the $\mathrm{O}_{2}$ molecule has a zero electric-dipole moment, the usual and prominent dipole-dipole or dipole-quadrupole interactions do not play any role [2]. The $\mathrm{O}_{2}$ molecule has a little quadrupole moment which gives a quadrupole-quadrupole interaction. In addition, other types of interactions can be present as, for example, polarizability interactions that can produce a line broadening comparable with that originated by the quadrupole force [2]. Of course, there is also the magnetic-dipole interaction, but this is found to be negligible [3].

$\left.{ }^{*}\right)$ The authors of this paper have agreed to not receive the proofs for correction.

(**) Permanent address: Facoltà di Scienze Ambientali della Seconda Università di Napoli, Via Arena 22, 81100 Caserta, Italy.

(***) Permanent address: European Laboratory for Non linear Spectroscopy (LENS), Largo E. Fermi 2, 50125 Firenze, Italy, 
In order to better understand the collisional mechanisms, systematic studies on pressure broadening and its dependence on temperature and rotational quantum numbers assume a great relevance.

These investigations have been mainly performed in the microwave region. In an early work on the magnetic-resonance spectrum of oxygen [4], the width of some lines at room and liquid-air temperatures was measured: the experimental data did not agree with a temperature dependence of $T^{-0.75}$ which was expected in the case of a pure quadrupole-quadrupole interaction. Nearly in the same period, the variation of linebreadth with the rotational quantum number $K$, at room temperature, was studied [2]; the analysis of the results suggested, besides quadrupole-quadrupole and polarizability interactions, contributions from rotational resonance interactions. For transitions in the same region, the nitrogen and self-broadening parameter was measured at three different temperatures [5]; the functional dependence, which was found to be intermediate between $T^{-0.7}$ and $T^{-2}$, was qualitatively explained introducing an additional contribution to the Lorentzian linewidth coming from resonant exchange processes.

Recently, a systematic study of the self-broadening coefficient as a function of the rotational quantum number has been performed in the near-infrared region [6], where molecular oxygen exhibits very weak absorption bands, corresponding to the $b^{1} \Sigma_{\mathrm{g}}^{+} \leftarrow X^{3} \Sigma_{\mathrm{g}}^{-}$electronic transition [7]; in particular, the $0-0$ vibrational band, which is designated by astronomers as atmospheric-oxygen $A$-band, was investigated at room temperature. Such a band, centred at $762 \mathrm{~nm}$, is extremely interesting in atmospheric remote-sensing applications, since it lies in a relatively empty portion of the atmospheric spectrum; as an example, lidar measurements of temperature, pressure and density are possible using the $A$-band, as demonstrated in ref. [8]. Such measurements need accurate information on the pressure and temperature dependence of the linewidth.

The $A$-band has been studied since 1927 [9]: using the Sun as a light source, the absorption spectrum in the atmosphere was observed. Individual lines occur as magnetic-dipole transitions; for this reason, the absorption coefficient is only $10^{-6} \mathrm{~cm}^{-1}$.

In this paper, we report a systematic study of collision linewidth as a function of temperature and pressure for the ${ }^{P} P(9)$ line of the oxygen $A$-band. Line profiles were observed with absorption spectroscopy technique, using a CW tunable diode laser. Thanks to the very low-amplitude noise level of the diode laser, high sensitivity was achieved and the investigation of the forbidden $A$-band was possible.

The temperature dependence of the pressure-broadening factor, which we found, was the commonly used $T^{-a}$ function, with the exponent $\alpha$ equal to $1.02 \pm 0.05$; it probably indicates that a contribution to Lorentzian linewidth coming from resonant exchange process is present.

Our data represent the first systematic investigation of the self-broadening parameter as a function of temperature in the molecular-oxygen spectrum.

\section{2. - Experimental details}

We used a commercial diode laser (Sharp LT024MD) that emitted a single-mode radiation at $780 \mathrm{~nm}$. Laser emission at $760 \mathrm{~nm}$ was achieved by cooling our device down to $190 \mathrm{~K}$; the injection current was $46 \mathrm{~mA}$. The cooling system was a 


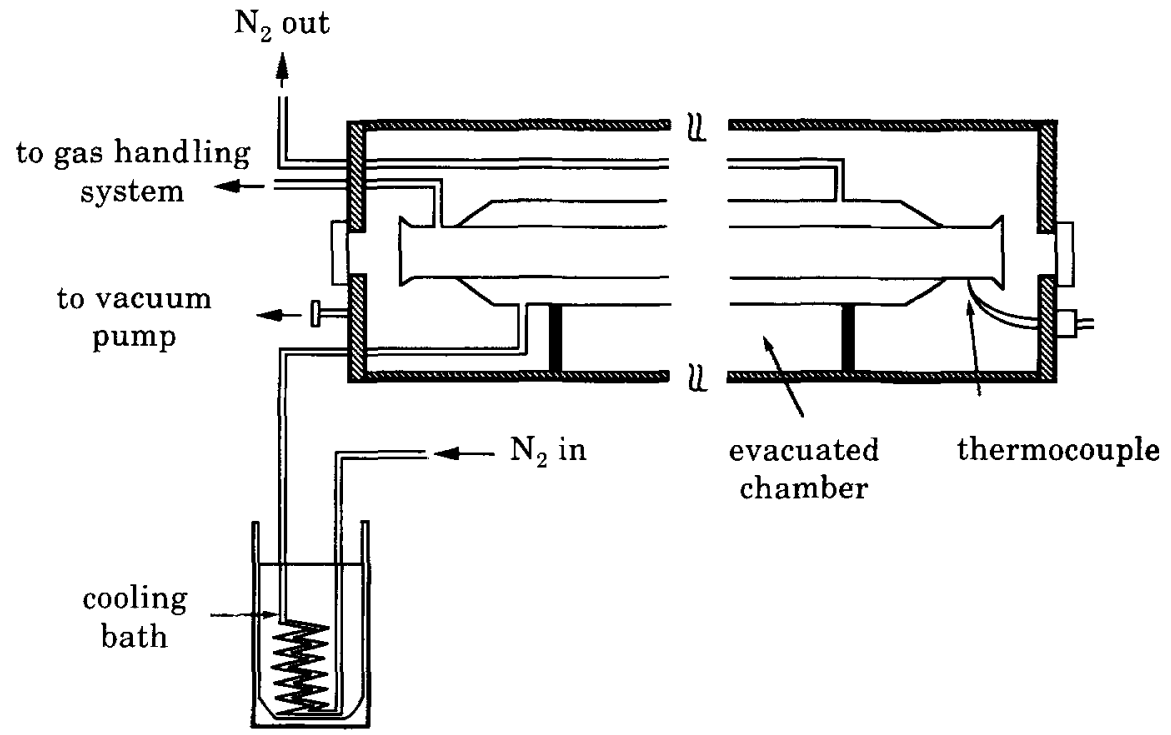

Fig. 1. - Details of our absorption cell. A nitrogen flow, which passes in a coil immersed in a cooling bath, is used to cool our cell from room temperature down to liquid-nitrogen temperature.

Joule-Thomson refrigerator. It is based on the expansion of a high-pressure nitrogen flow in a little chamber which is thermically connected with the diode laser. The diode was mounted inside a vacuum box and its temperature was actively stabilized to $10^{-2} \mathrm{~K}$ through a controller which used a temperature sensor and a heater. The laser emission bandwidth was measured to be $50 \mathrm{MHz}$ (FWHM) at the operating condition while the beam intensity was about $20 \mathrm{~mW}$. A low-noise current source was used as power supply for the diode. The laser light was collected and collimated by an AR-coated collimator, with a focal length of $14.5 \mathrm{~mm}$. A Faraday isolator was mounted immediately after the collimator, in order to avoid optical feedback.

The laser beam was splitted by a $50 \%$ beam splitter into two parts; one beam passed through the cell, while the second beam provided a reference signal. The absorption signal was subtracted from the reference one; in this way, the amplitude modulation originated from the diode laser, as the frequency was scanned, was cancelled. A fine balance between the intensity of the two beams was achieved using a light attenuator. The two beams had the same pathlength in air so that the background signal, coming from $\mathrm{O}_{2}$ absorption in air, was eliminated.

The minimum pressure, at which we could detect $\mathrm{O}_{2}$ absorption was 0.5 mbar.

The sample cell was a 1 meter long pyrex tube with a diameter of $2 \mathrm{~cm}$.

The cell was contained in a stainless-steel vacuum chamber in order to obtain thermal insulation and to avoid the water condensation on the end windows. The gas temperature was changed by means of a nitrogen flow circulating in a jacket surrounding the cell and previously cooled in a coil which was immersed in a freezing substance.

Different temperatures were achieved by changing the $\mathrm{N}_{2}$ flow and the freezing compound. We covered the entire range from room temperature to liquid-nitrogen 
$\left(\mathrm{LN}_{2}\right)$ temperature by using, as freezing substances dry ice, $\mathrm{LN}_{2}$ or mixtures of $\mathrm{LN}_{2}$ and acetone.

The details of our absorption cell and vacuum chamber are shown in fig. 1 .

The temperature of the system was controlled at two different points: on the jacket surrounding the cell and on the output of the $\mathrm{N}_{2}$ flow. However, a «real» measurement of the gas temperature was performed through a Voigt fit of the lineshapes obtained at low pressure (about 1 mbar), as will be discussed later.

The pressure inside the cell was measured with an $\mathrm{O}_{2}$-calibrated Pirani gauge.

The laser frequency was monitored by a Michelson wavemetre with an accuracy $\Delta v / v$ of $10^{-7}$.

Fine-frequency scans of few $\mathrm{GHz}$ across the selected line were obtained by tuning the diode injection current and were calibrated by means of a Fabry-Perot interferometer with a free spectral range of $1.5 \mathrm{GHz}$. Little non-linearity in the laser frequency scans observed in our recordings was corrected, by using an appropriate numerical analysis of the experimental data.

\section{3. - Results and discussion}

We investigated the magnetic dipole ${ }^{P} P(K=9)$ line at $13094.75 \mathrm{~cm}^{-1}$ of the $0-0$ band of the $b^{1} \Sigma_{\mathrm{g}}^{+} \leftarrow X^{3} \Sigma_{\mathrm{g}}^{-}$electronic transition. Line profiles at different temperatures and pressures were recorded. The temperature was changed between $77 \mathrm{~K}$ and $294 \mathrm{~K}$ and for each temperature, the $\mathrm{O}_{2}$ pressure varied from 1 bar down to few mbar. Each recorded profile was fitted with a Voigt function. However, a more appropriate profile is represented by the Galatry function which takes into account also Dicke narrowing effects. Nevertheless, using a Voigt function, we found deviations from observed profiles less than $1 \%$, which were negligible, in our case. Indeed, such deviations introduced an uncertainty on the pressure broadening coefficient which could be neglected with respect to other sources of uncertainty, as the oxygen pressure measurements. A fitted spectra is shown, as an example, in fig. 2.

The recording obtained at low pressure ( $P<5$ mbar) gave us information on the temperature inside the cell. In this case, the pressure broadening was neglected; so, in the fit programme, the homogeneous linewidth was kept fixed at the value of the laser linewidth while the Gaussian width was extrapolated. This procedure was used for each temperature, except for room temperature, which was directly measured with the thermocouple located on the cell.

In all other fits, obtained at pressures greater than 5 mbar, the Doppler width was fixed at the value observed in the low-pressure regime, at the same temperature, while the Lorentzian width, the line position, the line strength and the baseline were varied.

The Lorentzian linewidth (HWHM) at $T=209 \mathrm{~K}$, is plotted as a function of the $\mathrm{O}_{2}$ pressure in fig. 3.

The pressure-broadened Lorentzian linewidth is given by [1]

$$
\gamma(p, T)=\gamma(T) p .
$$

For each temperature $T$, a linear fit of the linewidth $v$ s. pressure data allows to obtain the pressure broadening coefficient $\gamma(T)$. These results are reported in table I and plotted in fig. 4. 


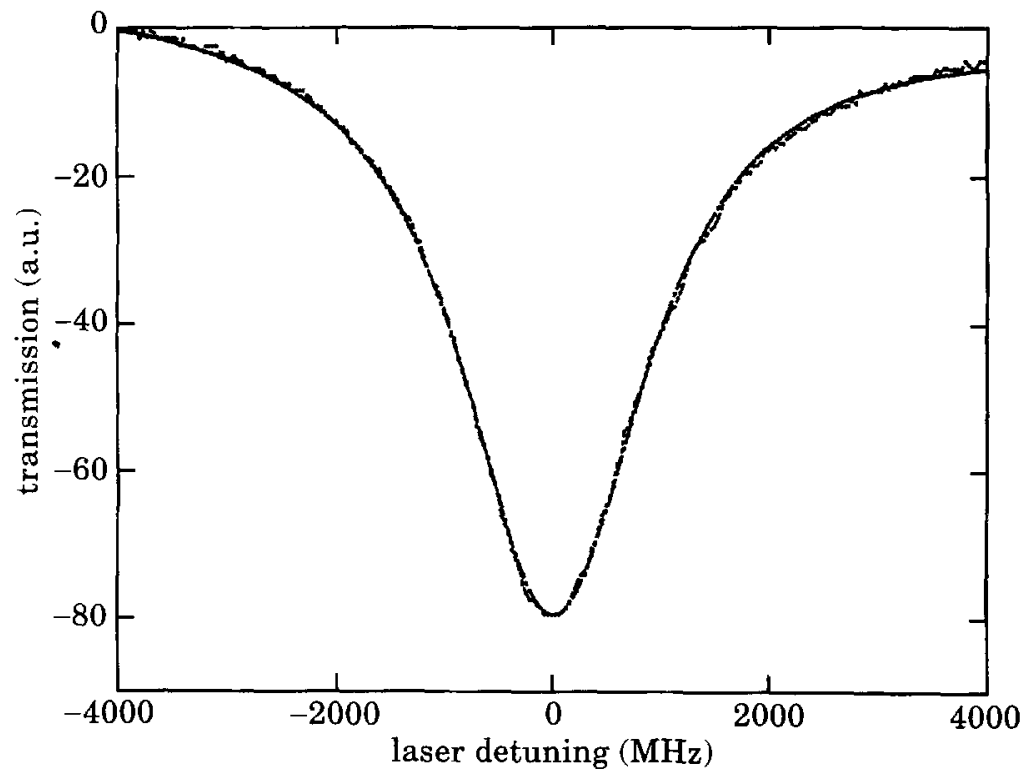

Fig. 2. - Example of an absorption spectrum $(P=127$ Torr and $T=78 \mathrm{~K})$; the continuous line represents the fit of the lineshape with a Voigt function.

According to theory, in the limit of validity of impact approximation, the pressure broadening coefficient has a $T^{-\alpha}$ dependence on temperature; considering an intermolecular potential proportional to $1 / r^{n}$, if the effect of inelastic collisions is neglected, the exponent $\alpha$ is given by [1]

$$
\alpha=(n+1) /[2(n-1)],
$$

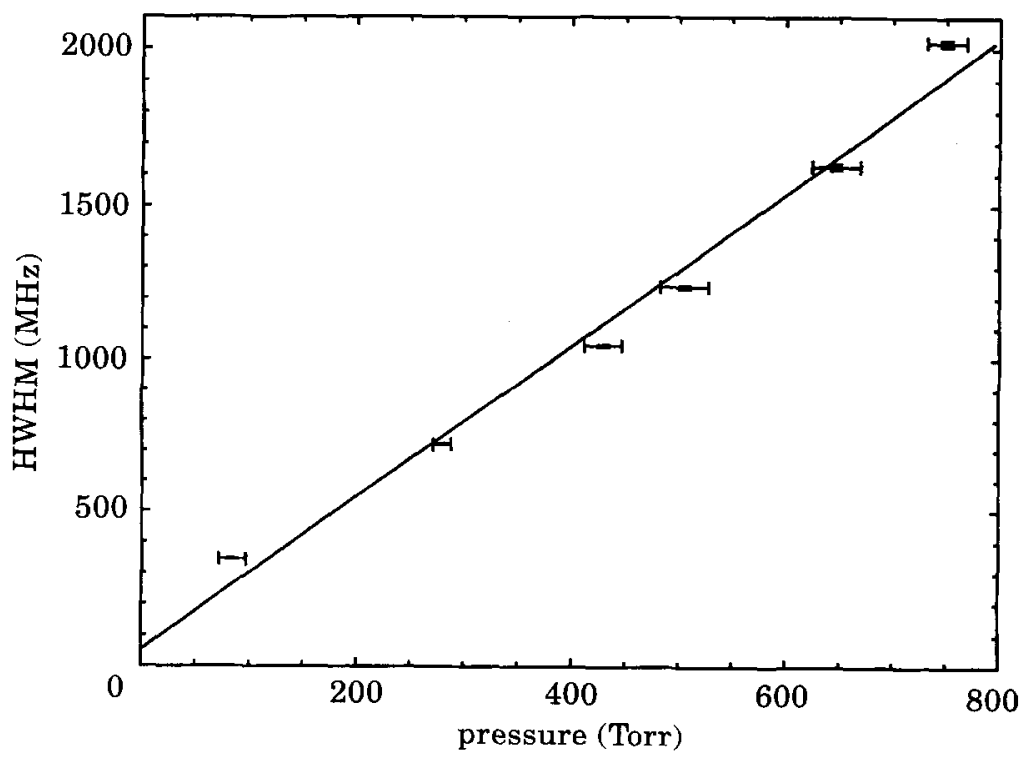

Fig. 3. - The Lorentzian linewidth as a function of pressure, at a gas temperature of $209 \mathrm{~K}$. 
TABLE I. - Measured pressure broadening coefficients at different gas temperatures.

\begin{tabular}{ll}
$\begin{array}{l}\text { Temperature } \\
(\mathrm{K})\end{array}$ & $\begin{array}{l}\text { Pressure broadening coefficient } \\
\text { (MHz/Torr) }\end{array}$ \\
\hline $292.25(0.01)$ & $3.75(0.09)$ \\
$231(6)$ & $4.78(1.15)$ \\
$209(6)$ & $4.92(0.12)$ \\
$132(6)$ & $8.1(0.2)$ \\
$93(6)$ & $11.7(1.4)$ \\
$78(1)$ & $14.8(1.1)$ \\
\hline
\end{tabular}

where $\alpha$ ranges between 0.5 and 1 . The 0.5 value is obtained in the extreme case of a temperature-independent collision cross-section, while $\alpha=1$ occurs when a dipoledipole interaction $(n=3)$ dominates.

In the case of molecular oxygen, quadrupole-quadrupole and polarizability interaction, proportional, respectively, to $r^{-5}$ and $r^{-6}$, are responsible of the selfbroadening. In this case, according to eq. (2), a $T^{-0.75}$ or $T^{-0.7}$ dependence of the self-broadening coefficient should occur.

Nevertheless, if the power law is quite well verified in most cases, different $\alpha$ values, with respect to that predicted by eq. (2), may be observed. In particular, considering the collisional energy transfer between internal degrees of freedom to translation, lower $n$ values are expected [10]. In addition, it has been demonstrated that the resonant exchange process can produce very different values of the exponent $\alpha[11]$. A resonant process occurs when the collisional change of the internal energy of the absorbing molecule is equal to that of the perturbing molecule so that no energy is transferred to translation.

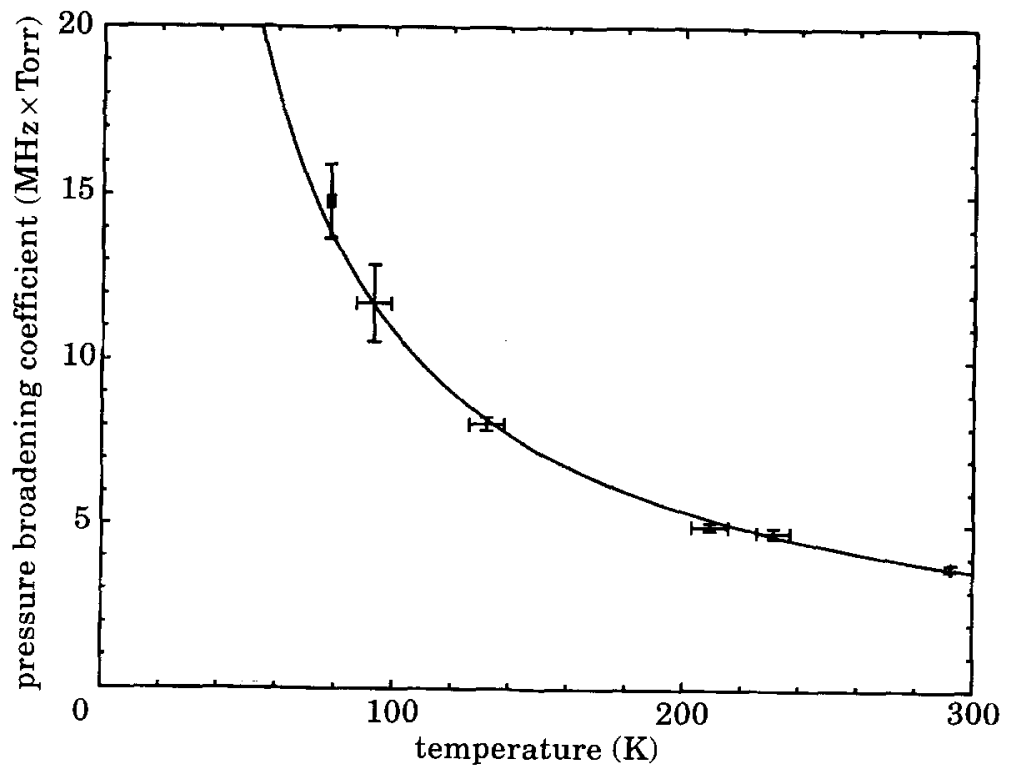

Fig. 4. - Pressure broadening coefficient vs. temperature; a $T^{-\alpha}$ dependence is observed. 
For instance, in the case of the self-broadening of $\mathrm{CH}_{3} \mathrm{CN}$ rotational spectrum, an exponent $\alpha>1$ for some lines and $\alpha<0$ for other lines has been measured [11].

In our case, by fitting the data reported in table I with a $T^{-a}$ function, we obtain

$$
\alpha=1.02 \pm 0.05 \text {. }
$$

This value indicates that other processes must contribute to the collisional broadening mechanisms.

The resonant exchange effect could explain the observed temperature dependence. The resonant process should play a role mainly in the electronic ground state, since the rotational levels of the $X^{3} \Sigma_{\mathrm{g}}^{-}(v=0)$ state are thermally populated. The interaction which couples the states involved in the exchange process can be the magnetic dipole or the quadrupole interaction. In order to test such hypothesis theoretical calculations are necessary. Furthermore, a wider experimental investigation is required; in particular, the temperature dependence of the collisional parameter could be repeated for other lines of the same band.

For this reason, we are going to use DFB (Distributed Feed-Back) diode lasers, emitting in a single mode at $760 \mathrm{~nm}$ at room temperature, which offer the advantage of a continuous and wider wavelength tunability with temperature, and also allow us to improve spectral resolution of the experimental apparatus.

\section{4. - Conclusion}

We observed the ${ }^{P} P(9)$ line of the electronic magnetic-dipole transition $b^{1} \Sigma_{\mathrm{g}}^{+} \leftarrow X^{3} \Sigma_{\mathrm{g}}^{-}$of molecular oxygen, using diode laser absorption spectroscopy technique. We studied the collisional broadening as a function of temperature and pressure. The temperature dependence of the self-broadening coefficient was measured to be $T^{-(1.02 \pm 0.05)}$. Our results could represent an experimental evidence of the resonant exchange process in the near-infrared spectrum of molecular oxygen.

The authors express their gratitude to G. Buffa for the stimulating discussion on pressure broadening theory and for sending us some preprints. We are also grateful to $\mathrm{M}$. Inguscio for his suggestions and encouragements, during this work.

[1] Townes C. H. and Schawlow A. L., Microwave Spectroscopy (Dover, New York, N.Y.) 1955.

[2] Anderson R. S., Smith W. V. and Gordy W., Phys. Rev., 87 (1952) 561.

[3] Mizushima M., Phys. Rev., 83 (1951) 94.

[4] Beringer R. and Castle J. G., Phys. Rev., 81 (1951) 1.

[5] Pickett H. M., Cohen E. A. and Brinza D., Astrophys. J., 248 (1981) L49.

[6] RitTer K. J. and Wilkerson T. D., J. Mol. Spectrosc., 121 (1987) 1. 
[7] Babcock H. D. and Herzberg L., Astrophys. J., 108 (1948) 167.

[8] Mason J. B., Appl. Opt., 14 (1975) 76.

[9] Dieke G. H. and BabCock H. D., Proc. Nat. Acad. Sci. USA, 13 (1927) 670.

[10] Baldacchini G., Ciucci A., D’Amato F., Buffa G. and Tarrini O., J. Quantum Spectrosc. Radiat. Trans, in press.

[11] Buffa G., Tarrini O., De Natale P., Inguscio M., Pavone F. S., Prevedelli M., Evenson K. M., Zink L. R. and SchwaAB G. W., Phys. Rev. A, 45 (1992) 6443. 